\title{
The axhs1/dwf4 auxin-hypersensitive mutant of Arabidopsis thaliana defines a link for integration of auxin and brassinosteroid mediated root elongation
}

\author{
Taisuke Nagase, Hisabumi Takase, Jiro Sekiya, Rafael Prieto* \\ Department of Bioscience and Biotechnology, Faculty of Bioenvironmental Science, Kyoto Gakuen University, Kameoka, \\ Kyoto 621-8555, Japan \\ *E-mail: raprieto@kyotogakuen.ac.jp Tel: +81-771-29-3493Fax: +81-771-29-3429
}

Received December 29, 2014; accepted February 9, 2015 (Edited by M. Sekine)

\begin{abstract}
To understand the molecular mechanism of auxin-mediated root elongation, we carried out screening to isolate auxin-hypersensitive (axhs) mutants. The T-DNA insertional mutant axhs1 was selected on the basis of root phenotypes associated with auxin sensitivity. Results from brassinosteroid (BR) feeding experiments, Tail-PCR and genetic analyses indicate that AXHS1 encodes DWF4, which catalyzes the rate limiting step of BR biosynthesis. The axhs1 mutant shows increased sensitivity to indole-3-acetic acid (IAA), the synthetic auxin 2,4-dichlorophenoxyacetic acid (2,4-D), auxin transport inhibitors such as 2,3,5-triiodobenzoic acid (TIBA) and N-1-naphthylphthalamic acid (NPA), and the antiauxin p-chlorophenoxyisobutyric acid (PCIB) for the root elongation response. Analysis of the expression of the DR5:GUS and HS:AXR3NT-GUS reporter genes in wild type and axhs1 genotypes, and characterization of double mutants between axhs 1 and mutants affected in auxin biosynthesis (wei2-1), auxin transport (aux1-7, eir1-1) and auxin signal transduction (tir1-1, axr1-3, axr2-1) indicate that auxin hypersensitivity in axhs1 is mediated by the auxin-signaling pathway and an AUX1, EIR1/ PIN2 dependent auxin uptake.
\end{abstract}

Key words: Arabidopsis thaliana, auxin, brassinosteroid, hormone signaling crosstalk, root elongation.

The plant hormone auxin, typified by indole-3-acetic acid (IAA), has been implicated in the regulation of plant growth and developmental processes such as cell division and expansion, lateral root formation, apical dominance, phototropism and gravitropism (Woodward and Bartel 2005).

Biochemical and molecular genetics approaches, including the isolation and characterization of Arabidopsis mutants and the cloning of the corresponding genes, have been a valuable strategy for the elucidation of auxin biosynthesis, transport and signal transduction pathways. Most of the mutants isolated so far have been selected on the basis of root phenotypes associated with auxin and/or auxin transport inhibitors resistance. Many of those mutants display cross-resistance to hormones such as ethylene, cytokinin, or abscisic acid. These pleotropic phenotypes illustrate the existence of crosstalk between different hormone signaling pathways (Lau et al. 2008; Woodward and Bartel 2005).

The auxin-deficient mutant wei2.1 is a loss-of-function mutant of the ASA1 gene, which encodes the $\alpha$-subunit of a rate limiting enzyme of tryptophan biosynthesis, anthranilate synthase (Stepanova et al. 2005). Tryptophan has been shown to be the main precursor for IAA in plants (Mashiguchi et al. 2011; Woodward and Bartel 2005). Mutants analysis have clearly implicated two gene families, the triptophan amino transferase of Arabidopsis (TAA) family and the YUCCA (YUC) family of flavin monooxygenases, in auxin biosynthesis (Mashiguchi et al. 2011; Woodward and Bartel 2005). Mashiguchi et al. (2011) reported that the TAA family is involved in the production of indole-3-pyruvic acid (IPA) from tryptophan, and the YUC family functions in the conversion of IPA to IAA. IAA exists in two principal forms: a) free IAA, which is the bioactive form; and b) conjugated to sugars, myoinositol, amino acids or small peptides. Local concentrations of free IAA are regulated by the interplay of biosynthesis, formation of conjugates, hydrolysis of conjugates to free IAA, and transport (Woodward and Bartel 2005).

Several Arabidopsis mutants are defective in proteins mediating polar auxin transport (PAT). Among them,

Abbreviations: ACC, 1-amino-cyclopropane-1-carboxylic acid; ARF, auxin response factor; AXHS, auxin-hypersensive; BL, brassinolide; BR, brassinosteroid; Brz, brassinazole; GUS, $\beta$-glucuronidase; IAA, indole-3-acetic acid; NAA, 1-naphtaleneacetic acid; NPA, N-1-naphthylphthalamic acid; PCIB, p-chlorophenoxyisobutyric acid; TIBA, 2,3,5-triiodobenzoic acid; 2,4-D, 2,4-dichlorophenoxyacetic acid.

This article can be found at http://www.jspcmb.jp/

Published online March 28, 2015 
AUX1 mediates influx of IAA into cells (Marchant et al. 1999). In contrast, the PIN family of proteins is involved in polar auxin efflux (Woodward and Bartel 2005). The aux1 loss-of-function mutations confer agravitropic phenotype, and resistance to auxin and ethylene in roots (Marchant et al. 1999). In contrast, the eir1 loss-offunction mutants displayed agravitropic root growth and were less sensitive to ethylene inhibition than was root growth of wild type plants (Luschnig et al. 1998). AUX1 plays an important role in regulating the hormone flux between IAA source and sink tissues, thereby influencing lateral root development and root gravitropic responses (Marchant et al. 1999, 2002; Swarup et al. 2001). The asymmetric localization of AUX1 and the auxin efflux transporter PIN1 to the upper and lower plasma membranes of protophloem cells has been proposed to promote the acropetal, post-phloem transport of auxin to the root apex (Swarup et al. 2001). On the other hand, it has been proposed that AUX1 and PIN2/EIR1 regulate root gravitropism by facilitating basipetal redistribution of auxin from the columella to distal elongation zone tissues via lateral root cap and epidermal tissues (Muller et al. 1998; Swarup et al. 2001). The polarity of basipetal transport in root is likely to be regulated by the apical plasma membrane localization of PIN2 in epidermal cells of the meristematic and elongation zones (Muller et al. 1998; Rahman et al. 2007). In contrast, the lack of polar localization of AUX1 in plasma membranes of lateral root cap and epidermal cells suggests that AUX1 does not actively direct the basipetal transport of auxin in roots (Swarup et al. 2001, 2004). On the other hand, PIN2 has a basipetal localization in cortical cells of the root elongation zone, and an acropetal localization in cortical cells of the meristem (Rahman et al. 2007). It is noteworthy to indicate that polar localization of PIN2 in cortical root cells has been proposed to represent a mechanism for fine-tuning the flow of auxin as required for optimal gravitropic responses (Rahman et al. 2010). Besides PIN proteins, multidrug resistance-like ABC transporters play also an important role in both acropetal and basipetal auxin transport (Wu et al. 2007).

The mechanism of auxin perception to transcriptional response acts trough alleviation of transcriptional repression. The current model proposes that under basal auxin conditions, a family of transcriptional repressors called AUX/IAAs bind to specific member of a family of transcriptional factors (ARFs), which show either repressor or activator activity. Increased auxin level facilitates the binding of the hormone to the F-box protein TIR1, a component of the E3 ubiquitin ligase complex $\mathrm{SCF}^{\mathrm{TIR} 1}$, which triggers the ubiquitin-mediated proteolysis of AUX/IAA proteins. The destruction of AUX/IAA allows the release of ARFs and thereby triggers the auxin response (Lau et al. 2008; Ruegger et al. 2012; Woodward and Bartel 2005). The AXR1 gene encodes a protein related to the ubiquitin-activating enzyme (E1), the first enzyme in the ubiquitin conjugation pathway (Leyser et al. 1993). The axr1 loss-of-function mutations confer auxin resistance to the root, and a variety of morphological defects including decreases in plant height, hypocotyl and stem elongation, irregular rosette leaves, and inflorescences more highly branched (Leyser et al. 1993). The semidominant tir1-1 mutant displays increased root growth on medium containing either auxins or auxin transport inhibitors (Ruegger et al. 2012). On the other hand, dominant gain-of-function mutations in AXR2 (AUX/IAA17) cause auxin and ethylene-resistant root growth, agravitropic root growth, a short hypocotyl and stem, and rosette leaves are small, round, and have a short petiole (Nagpal et al. 2000). Antiauxins such as p-chlorophenoxyisobutyric acid (PCIB) and yokonolide $\mathrm{B}$ (Ykb) inhibit the $\mathrm{SCF}^{\mathrm{TIR} 1}$ mediated auxin degradation of AUX/IAA proteins (Hayashi et al. 2003; Oono et al. 2003).

Microarrays analyses indicated that previously characterized auxin-responsive genes are not properly regulated when brassinosteroid (BR) biosynthesis is inhibited by brassinazole (Brz) (Chung et al. 2011). Besides, it has been published that the Auxin Response Element (AuxRE, TGTCTC) is enriched in genes regulated by both auxin and $B R$, which suggests that $\mathrm{BRs}$ and auxin signals converge at ARFs to regulate gene expression (Goda et al. 2004; Nemhauser et al. 2004). BRs induce early auxin-inducible genes (IAA, SAUR$A C 1$ ) and DR5:GUS without increasing the endogenous auxin levels per gram fresh weight (Nakamura et al. 2003a, b). The levels of the IAA5, IAA19 transcripts were higher in wild type than in the BR-deficient mutant $\operatorname{det} 2$, even though IAA levels per gram fresh weight were lower in wild type seedlings (Nakamura et al. 2003a). AUX/IAA7 (AXR2) and AUX/IAA17 (AXR3) proteins are required for full BR induction of IAA5 and IAA9, but they do not have a significant role in BR-mediated induction of SAUR-AC1 (Nakamura et al. 2006). Besides, Nakamura et al. (2006) reported that Ykb inhibited both auxin, and BR-induced expression of IAA5 and SAUR$A C 1$, whereas PCIB only inhibited the auxin-induced expression of those genes. Based on these results, it was proposed that BR and auxin signaling pathways crosstalk downstream of auxin, somewhere between the site of PCIB and Ykb actions (Nakamura et al. 2006).

This work presents genetics and molecular evidences about the cross talk between BR and auxin during Arabidopsis root elongation. In order to broaden the spectrum of auxin mutants, we conducted a screen for auxin-hypersensitive (axhs) mutants selected on the basis of root phenotypes associated with auxin sensitivity. Characterization of the T-DNA insertional mutant axhs1 indicates that AXHS1 encodes DWF4, the rate limiting enzyme of BR biosynthesis (Choe et al. 1998). The axhs1 
mutant showed increased sensitivity to IAA, the synthetic auxin 2,4-dichlorophenoxyaceticacid (2,4-D), auxin transport inhibitors such as 2,3,5-triiodobenzoic acid (TIBA) and N-1-naphthylphthalamic acid (NPA), and PCIB. Genetic analyses of the interaction between the axhs 1 mutation and mutations affecting genes involved in auxin biosynthesis (wei2-1), auxin transport (aux1-7, eir 1-1) and auxin signal transduction (tir1-1, axr1-3, axr2-1) indicate that auxin transport and functional components of the auxin-signaling pathway are required for the auxin-hypersensitive phenotype of axhs1. In addition, analysis of the expression of the DR5:GUS and HS:AXR3NT-GUS reporter genes in wild type and axhs 1 genotypes indicates that the axhs 1 mutation alters auxin responsiveness.

\section{Materials and methods}

\section{Plant materials and growth conditions}

The $d w f 4-102$ (SALK_020761), $d w f 4-103$ (SAIL_882_F07), tir1-1, axr1-3, axr2-1, eir1-1, aux1-7, and wei2-1 mutants, DR5:GUS and HS:AXR3NT-GUS transgenic lines were obtained from the Arabidopsis Biological Resource Center (ABRC) at Madison, USA.

A. thaliana seeds were surface sterilized in a mixture of $17 \%$ sodium hypochlorite $(\mathrm{v} / \mathrm{v})$ and $4 \%$ triton-X (v/v), and were allowed to germinate on plates containing Arabidopsis nutrient solution (Haughn and Somerville 1986), 1.5\% sucrose and $1 \%$ agar. Plates were routinely kept in the dark for 2 days at $4^{\circ} \mathrm{C}$ to break seed dormancy, and then incubated in a near vertical position at $23^{\circ} \mathrm{C}$ with a $16 \mathrm{~h}$ light $/ 8 \mathrm{~h}$ dark cycle. Unless otherwise indicated, seedlings were grown under light conditions. For dark conditions, plates were wrapped in three layers of aluminum foil. Day 0 of grow is defined as the time when plates were transferred to $23^{\circ} \mathrm{C}$. Plants were grown to maturity on metromix 350 .

\section{Growth assays}

Seedlings were germinated and grown for 7 days on solid media supplemented with or without the corresponding growth regulators. Final concentration of IAA, 2,4-D, and NAA ranged from 30 to $180 \mathrm{nM}$; BR concentration ranged from 0.1 to $10 \mathrm{nM}$; TIBA, and NPA concentration was 2,5 and $5 \mu \mathrm{M}$; and PCIB concentration ranged from 5 to $15 \mu \mathrm{M}$. Lengths of the root and hypocotyl of at least 20 seedlings were measured with a graduated ruler.

\section{Isolation of axhs T-DNA insertion mutants}

The axhs1 mutant was isolated from an Arabidopsis transgenic plant collection (ecotype Columbia, Col, Alonso et al. 2003) available at the ABRC.

Thermal asymmetric interlaced (Tail-) PCR cloning of the AXHS1 gene

Tail-PCR analysis was performed as described by Liu et al. (1995). The T-DNA insertion point was determined by sequencing the putative T-DNA-AXHS1 tertiary PCR products identified in the analysis.

\section{RNA isolation and RT-PCR analysis}

Total RNA was isolated using RNeasy mini kit (Qiagen, Germany) following manufacturer's instructions. First strand cDNA was synthesized from about $1 \mu \mathrm{g}$ total RNA from 7-day-old Arabidopsis seedlings, using PrimeScriptTM first strand cDNA synthesis kit (Takara, Japan) as described by the manufacturer. PCR was carried out under standard conditions, using axhs5-3 (5'-AGC ACT CAA AGA TGT TCG GTA CA-3') and axhs3-6 (5' -CCC TAA TAG GCA AAC CGT TAG GA-3') primers, which leads to the amplification of a $365 \mathrm{bp}$ DWF4 a cDNA fragment. A $516 \mathrm{bp}$ AUX1 cDNA fragment, and a $422 \mathrm{bp}$ PIN2/EIR1 cDNA fragment were amplified using primer sets AUX1 (5' -ATG TCG GAA GGA GTA GAA GCG A-3' and $5^{\prime}$-GTT CTC TTG TCC AGA TGA TCG T-3'), and PIN2/ EIR1 (5' -ATG ATC ACC GGC AAA GACATG T- $3^{\prime}$ and $5^{\prime}{ }^{\prime}-$ CTC TGA AGC ACC ACG ATC TGC A-3' $)$, respectively. The primer set Act2 (5'-GTT GGT GAT GAA GCA CAA-3' and 5' -CAA GAC TTC TGG GCA TCT-3') was used to amplify a $425 \mathrm{bp}$ fragment of Actin2 cDNA as an internal standard of gene expression.

\section{Genetic crosses}

Double mutants were selected in F2 populations by genotyping analysis, and the homozygous double mutants in the F3 generation were used for phenotypic analysis. GUS reporters (DR5:GUS, HS:AXR3NT-GUS) were introduced into the axhs1 mutant background by crossing.

The genotype of the axhs1-1 mutant was confirmed by genomic PCR analysis using the T-DNA left border primer LBa 1 (5' - TGG TTC ACG TAG TGG GCC ATC G-3') and AUXS1/DWF4 specific primers axhs5-3 and axhs3-3 (5'-AGC ACT CAA AGA TGT TCGGTA CA- $\left.3^{\prime}\right)$. The presence of the GUS transgene was verified using gusF $\left(5^{\prime}\right.$-ATC GTG CTG CGT TTC GAT GCG GT-3') and gusR (5'-AGG TTA AAG CCG ACA GCA GCA GT- $3^{\prime}$ ) as GUS specific primers. The wei2-1, axr1-3, axr2-1, tir1-1, eir1-1, and aux1-7 mutations were analyzed by using cleaved amplified polymorphic sequence (CAPS) markers. The $269 \mathrm{bp}$ PCR product amplified from wei2-1 with wei2F ( $5^{\prime}$-GAA TCC AAG TCC GTA TAT GGG TTA TTT CTA G-3') and wei2R (5'-CGA TTC ACT ATC TTG TTC TGC TTC A-3'), and the $212 \mathrm{bp}$ fragment amplified from aux 1-7 with aux $1 \mathrm{~F}$ ( $5^{\prime}$-ACC AAC TTT GTT CGT CAA GTC GAC ACT CTA G-3') and aux1R (5'-AGC ACG CAT TTA AAG GGG TGT GT-3') were digested by $\mathrm{XbaI}$ in wei2-1 and aux1-7, but they were not cleaved in wild type genotypes. On the other hand, there was an HaeIII site in the $204 \mathrm{bp}$ PCR product amplified from wild type DNA with axr2F (5' -ACA TGCGTA CAA GCA AAC ATG A-3') and $\operatorname{axr} 2 \mathrm{R}$ (5'-CAC CAC TAC TGG TCT TCT GCT GA-3') primers, an NdeI site in the $209 \mathrm{bp}$ fragment amplified from wild type DNA with axr1F (5'-GGTGGA AGA TTC AAT GTT GAA ACT TGA TAG CAT AT- $\left.3^{\prime}\right)$ and axr1R (5'-GGCCAA TAT 
CCT AACCACGGCAT-3'), an NcoI site in the 231 bp PCR product amplified from wild type DNA with tir1F $\left(5^{\prime}\right.$-TCC TGC GAA GGC TTC TCC ACCCAT G- $3^{\prime}$ ) and tir1R (5'-GTA TAA TGA ATA TAC AAA TCA CAC ACC A-3'), and a Pst site in the $225 \mathrm{bp}$ fragment amplified from wild type DNA with eir1F (5' -TGC TTG ATG TTG TTG ATC ATT TTA TGC TGC A-3 $\left.{ }^{\prime}\right)$ and eir1R ( $5^{\prime}$-GCA ATA ATC TTT GGT TGC AAT GCC A-3'), that were absent in axr2-1, axr1-3, tir1-1, and eir1-1 genotypes, respectively.

\section{GUS expression analysis}

Seven day-after-germination DR5:GUS and axhs1 DR5:GUS seedlings were treated with growth regulators for $48 \mathrm{~h}$ and then incubated in GUS staining buffer containing $50 \mathrm{mM}$ potassium phosphate buffer ( $\mathrm{pH} 7), 20 \%$ (v/v) methanol, $10 \mathrm{mM}$ EDTA, $0.5 \mathrm{mM}$ potassium ferricyanide, $0.5 \mathrm{mM}$ potassium ferrocyanide, $0.1 \%(\mathrm{v} / \mathrm{v})$ Triton $\mathrm{X}-100$ and $1 \mathrm{mM}$ 5-bromo-4-chloro-3-indolyl- $\beta$-D-glucuronide (X-Gluc) at $25^{\circ} \mathrm{C}$ for $20 \mathrm{~h}$. Samples were then incubated sequentially in $90 \%$, $80 \%$, and $70 \%$ ethanol to remove plant pigments. After that, samples were whole mounted in chloral hydrate solution ( $8 \mathrm{~g}$ of chloral hydrate, $1 \mathrm{ml}$ of glycerol, and $2 \mathrm{ml}$ of water) to enhance transparency of the tissue.

\section{Protein stability assays}

Seven day-after-germination HS:AXR3NT-GUS and axhs1 $H S: A X R 3 N T-G U S$ seedlings were submerged into liquid medium and heat shocked for $2 \mathrm{~h}$ at $37^{\circ} \mathrm{C}$. After that, seedlings were transferred to fresh medium for $20 \mathrm{~min}$, treated for $10-$ 105 min in medium supplemented with growth regulators, and stained with $\mathrm{X}$-gluc for $20 \mathrm{~h}$ as described above.

\section{Results}

\section{Isolation of the axhs 1 mutant}

In order to identify new genes involved in auxin response we screened 3,300 T-DNA transformed lines of ecotype Columbia (Col) for seedlings with an increased sensitivity to 2,4-D for the root elongation response. One mutant line (axhs1) was selected as an auxin-hypersensitive mutant (Figure 1A). The roots of axhs1 seedlings grown in control medium were somewhat shorter than those of the wild type, but the main characteristic of the axhs1 mutant is a short hypocotyl in seedlings grown under both light and dark conditions (Figure 1A, B, Supplementary Figure S1). On the other hand, mature axhs1 plants showed a weak dwarf phenotype. The height of axhs1 plants was reduced compared to wild type (Figure 1C-E, Figure 6, Supplementary Figure S1). Besides, they exhibited round rosette leaves with short petioles (Figure 1C, D), and a large number of siliques which were smaller compared to those of wild type plants (Figure 1D, E, Supplementary Figure S1).

\section{Root elongation in axhs 1 is hypersensitive to auxins, antiauxins, and auxin transport inhibitors}

In order to characterize the response of axhs 1 to auxin and related compounds, wild type and axhs 1 seeds were germinated directly on growth medium supplemented with the compound of interest and root length was scored after 7 days. The results of the different assays are expressed as a percentage of growth in the absence of the

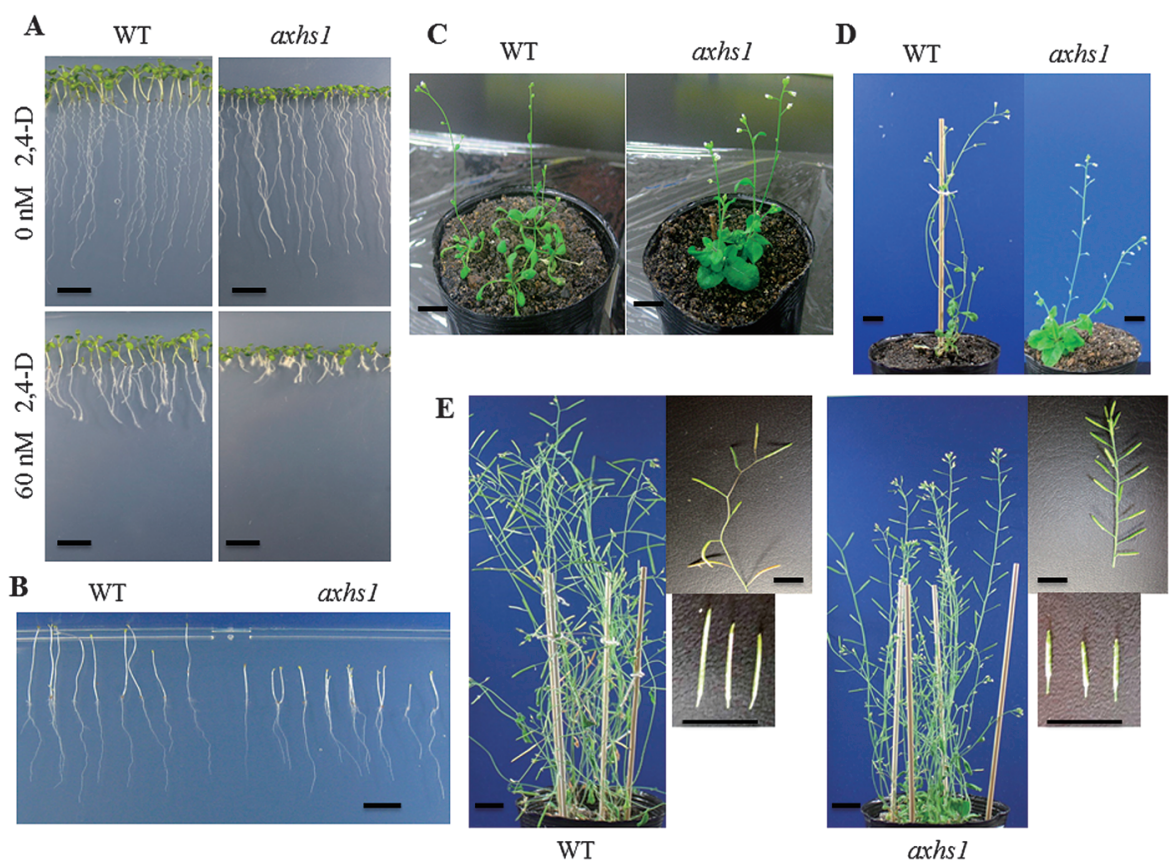

Figure 1. Comparison of the auxin sensitivity and morphology of wild type (Col) and axhs1 mutant plants. (A) Phenotypes of seedlings grown for 7 days on 0 and $60 \mathrm{nM}$ 2,4-D. (B) Seven-day-old etiolated seedlings. (C) Morphology of plants at 21 (C), 28 (D), and 44 days (E). Insets show a magnified view of siliques. Bars $=1 \mathrm{~cm}$ 


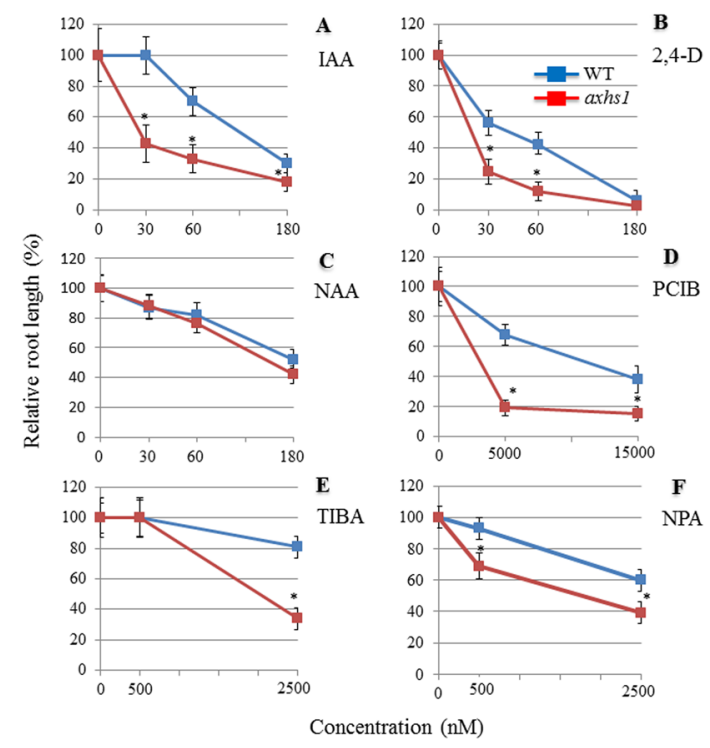

Figure 2. axhs1 is hypersensitive to auxins, antiauxins, and auxin transport inhibitors. Dose-response curves of roots of 7-day-old wild type and axhs 1 seedlings grown in solid medium supplemented with or without IAA (A), 2,4-D (B), NAA (C), PCIB (D), TIBA (E), NPA (F). Relative root length is expressed as a percentage of the length observed in unsupplemented medium. Values are means \pm SD of at least two independent experiments in which root length of 20-25 seedlings was measured. An asterisk indicates a significant difference from wild type by Student's $t$-test $(p<0.05)$.

corresponding compound. The axhs1 mutant showed increased sensitivity to IAA, the synthetic auxin 2,4-D, auxin transport inhibitors such as TIBA and NPA, and the antiauxin PCIB (Figure 2). In contrast, the roots of axhs1 showed will type sensitivities to the synthetic auxin NAA (Figure 2), the ethylene precursor (1-aminocyclopropane-1-carboxylic acid) ACC, and kinetin (data not shown). These results suggest that AXHS1 is involved in auxin transport and/or signal transduction.

\section{axh1 is a weak allele of DWF4}

Tail-PCR analysis indicated the presence of a T-DNA inserted in intron 7 of the DWF4 gene in axhs1 (Figure $3 \mathrm{~A})$. DWF4 encodes a C-22 hydroxylase that is crucial for BR-biosynthesis and the feedback control of endogenous BR levels (Choe et al. 1998; Yoshimitsu et al. 2011). Since the T-DNA insertion was found in intron 7 , the axhs1 gene might produce a wild type DWF4 protein and/or a non-functional protein lacking the conserved heme-binding domain (Figure 3A, B). The expression of DWF4 in axhs1 was analyzed by RT-PCR, using primers designed for amplification of a $365 \mathrm{bp}$ cDNA fragment including part of exon 6 and 8 (Figure 3A). RT-PCR analysis indicated that axhs 1 showed low levels of mature DWF4 mRNA accumulation compared to wild type (Figure 3C). The identity of spliced DWF4 cDNA was verified by cDNA sequencing.

We crossed axhs1 with wild type Col and analyzed 60 seedlings from the F2 generation. Results indicated that the axhs1 phenotypes, including auxin hypersensitivity and short hypocotyl (Figure 3D), were caused by a single recessive mutation linked to the T-DNA insertion in the DWF4 gene. In order to confirm that the axhs1 phenotypes are caused by a T-DNA insertion in DWF4 we analyzed other $d w f 4$ alleles of the Col ecotype. $d w f 4$ 102 is a strong mutant allele with a T-DNA inserted in exon 5 of DWF4 (Nakamoto et al. 2006). We searched for other $d w f 4$ alleles and found a line (SAIL_882_ $\mathrm{F} 07=d w f 4-103)$ carrying a T-DNA insert in intron 7 of DWF4 (Figure 3A). The phenotype of axhs1 and $d w f 4$ 103 plants was quite similar (data not shown), and clearly resembled that of weak alleles of DWF4 (Nakamoto et al. 2006). Moreover, as illustrated in Figure 3E, $d w f 4$ 102 and $d w f 4-103$ had a level of sensitivity to $2,4-\mathrm{D}$ similar to that of axhs1. On the other hand, we carried out a complementation test between axhs 1 and $d w f 4$ 102. Since strong mutant alleles of DWF4 are infertile (Azpiroz et al. 1998; Nakamoto et al. 2006), heterozygous dwf4-102 was crossed with axhs1. Analysis of the F1 and F2 segregation indicated that axhs1 is allelic to $d w f 4$ 102 (Figure 3F). Thus, hereafter, axhs1 will be named axhs $1 / d w f 4$.

In order to verify the cross talk between auxin and BR, we examined whether the short hypocotyl and auxin hypersensitivity of axhs1/dwf4 could be rescued by exogenous application of BR. As shown in Figure 4, the short hypocotyl of axhs1/dwf4 was partially rescued by addition of $10 \mathrm{nM}$ brassinolide (BL) (Figure 4A). On the other hand, $0.1 \mathrm{nM}$ BL promoted root growth in wild type and axhs1 seedlings grown in auxin-free medium (Figure 4B). Besides, the inhibition of root growth by 2,4-D in wild type and axhs1 seedlings was rescued by $\mathrm{BL}$ (Figure 4B). These results confirm that axhs $1 / d w f 4$ is a BR-deficient mutant, and suggest that BRs negatively regulate auxin sensitivity in roots.

\section{Genetic interaction between axhs1/dwf4 and auxin mutants}

Our data indicate that axhs1 is a $d w f 4$ mutant allele that is hypersensitive to auxins, the antiauxin PCIB and auxin transport inhibitors such as TIBA and NPA. In order to analyze the impact of the axhs1/dwf4 mutation on auxin biosynthesis, transport, and signaling, we constructed and characterized double mutants between axhs1/dwf4 and mutants affected in auxin biosynthesis (wei2-1), auxin transport (aux1-7, eir1-1), and auxin signal transduction (tir1-1, axr1-3, axr2-1). The level of sensitivity of wild type, single, and double mutant roots to IAA, 2,4-D, NPA and PCIB is illustrated in Figure 5.

It is noteworthy to indicate that the auxin-deficient mutant wei2.1 showed increased sensitivity to PCIB (Figure 5), which could be due to an altered ratio of the auxin-to-antiauxin signal in this mutant. The axhs 1 wei2-1 mutant exhibited levels of IAA, 2,4-D, NPA 

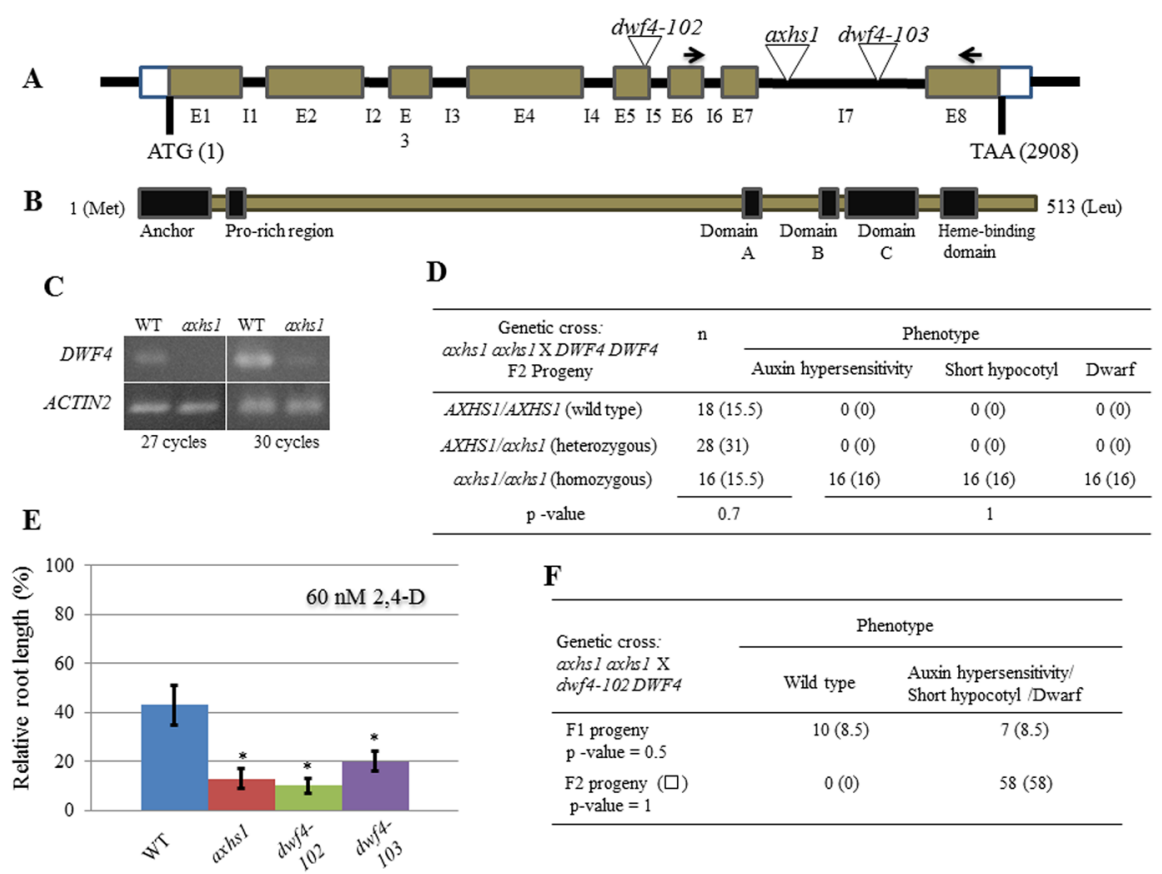

F

\begin{tabular}{lcc}
\hline & \multicolumn{2}{c}{ Phenotype } \\
\cline { 2 - 3 } $\begin{array}{l}\text { Genetic cross: } \\
\text { axhs 1 axhs } 1 \mathrm{X} \\
\text { awf4-102DWF4 }\end{array}$ & Wild type & $\begin{array}{l}\text { Auxin hypersensitivity/ } \\
\text { Short hypocotyl / Dwarf }\end{array}$ \\
\hline $\begin{array}{l}\text { F1 progeny } \\
\text { p -value }=0.5\end{array}$ & $10(8.5)$ & $7(8.5)$ \\
$\begin{array}{l}\text { F2 progeny ( } \\
\text { p-value }=1\end{array}$ & $0(0)$ & $58(58)$ \\
\hline
\end{tabular}

Figure 3. Tail-PCR cloning of AXHS1/DWF4: ahxs1 is a weak allele of DWF4 gene. (A) Genomic structure of the DWF4 gene. Rectangles and lines represent exons and introns, respectively. Triangles indicate the position of T-DNA insertions. Arrows indicate the primers used in RT-PCR expression analysis in (C). (B) Major domains of the DWF4 protein (Choe et al. 1998). (C) RT-PCR analysis. Seven-day-old wild type and axhs1 seedlings were analyzed for expression of DWF4. The number of PCR cycles is indicated. The figure illustrates representative results from three independent experiments. (D) F2 progeny from the cross between wild type and axhs1 was analyzed for axhs1 phenotypes. Auxin sensitivity was determined in seedlings grown in solid medium supplemented with $60 \mathrm{nM}$ 2,4-D for 7 days. Genotypes were verified by genomic PCR using axhs5-3, axhs3-3, and LBa1 primers. $\mathrm{p}=$ value of Chi-square test. The expected data, which are written in parenthesis, were calculated on the assumption that auxin hypersensitivity, short hypocotyl and dwarf phenotypes are caused by a single recessive mutation. $n=$ number of plants analyzed. (E) $d w f 4$ mutants are hypersensitive to auxin. Wild type, axhsl, $d w f 4-102$ and $d w f 4-103$ seedlings were grown for 7 days in solid medium supplemented with or without $60 \mathrm{nM}$ 2,4-D. Root length is expressed relative to untreated seedlings. Average and SD of more than 20 seedlings are shown. An asterisk indicates a significant difference from wild type by Student's $t$-test $(p<0.05)$. (F) Progeny from the cross between axhs 1 (homozygous) and $d w f 4-102$ (heterozygous) was evaluated for auxin sensitivity, short hypocotyl, and dwarf phenotypes as indicated in (C). $\square=$ The progeny from 4 independent F1 plants exhibiting auxin hypersensitivity, short hypocotyl, and dwarf phenotypes was analyzed. $\mathrm{p}=$ value of Chi-square test. The expected data, which are written in parenthesis, were calculated on the assumption that axhs 1 and $d w f 4-102$ are allelic mutations.
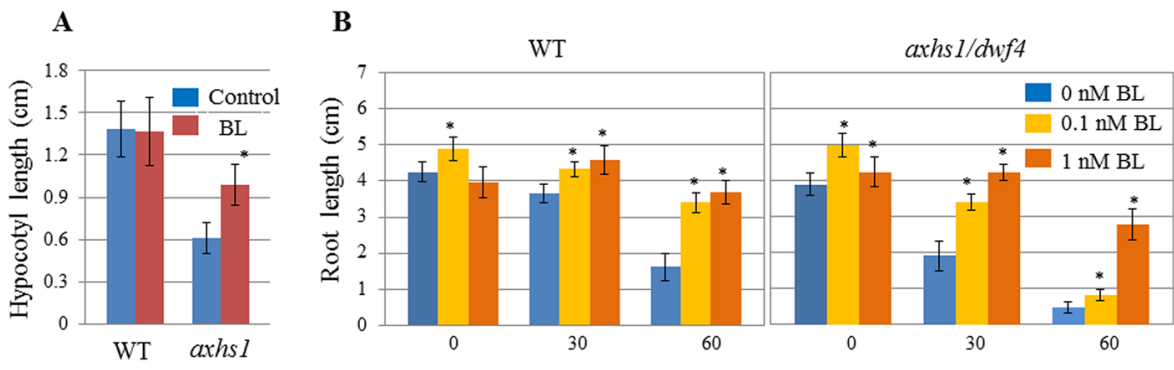

2,4-D concentration $(\mathrm{nM})$

Figure 4. The short hypocotyl and auxin hypersensitive phenotypes of ahxs 1 are rescued by BL. (A) Wild type and axhs1/dwf4 seedlings were grown for 7 days in the dark in medium supplemented with and without $10 \mathrm{nM} \mathrm{BL}$. (B) Wild type and axhs 1 seedlings were grown for 7 days on 0 , 30 , and $60 \mathrm{nM}$ 2,4-D medium supplemented with 0, 0.1, and $1 \mathrm{nM} \mathrm{BL}$. Average and SD of the root and hypocotyl length of more than 20 seedlings are shown. An asterisk indicates a significant difference from BL-free medium supplemented with 0,30, or $60 \mathrm{nM} 2,4$-D, by Student's $t$-test $(p<0.05)$.

and PCIB sensitivity (Figure 5) similar to those of the axhs1/dwf4 mutant. On the other hand, the morphology of the axhs1/dwf4 wei2-1 mutant was indistinguishable from that of the axhs1/dwf4 single mutant (Figure 6 and Supplementary Figure S2). These results strongly suggest that endogenous auxin levels have no significant impact on the axhs1/dwf4 mutant phenotypes. To further evaluate a possible role of BR in ASA1 (WEI2)-mediated auxin biosynthesis, the ASA1:GUS reporter gene (Stepanova et al. 2005) was introduced in the axhs1/dwf4 background by genetic crosses, and its expression was examined. The expression of ASA1:GUS was not affected by BL treatment (Supplementary Figure S3). Besides, no significant difference in GUS staining was observed 
between wild type and axhs1 seedlings treated with or without BL (Supplementary Figure S3).

The axr1-3 mutant showed increased resistance to NPA and PCIB compared to wild type seedlings (Figure

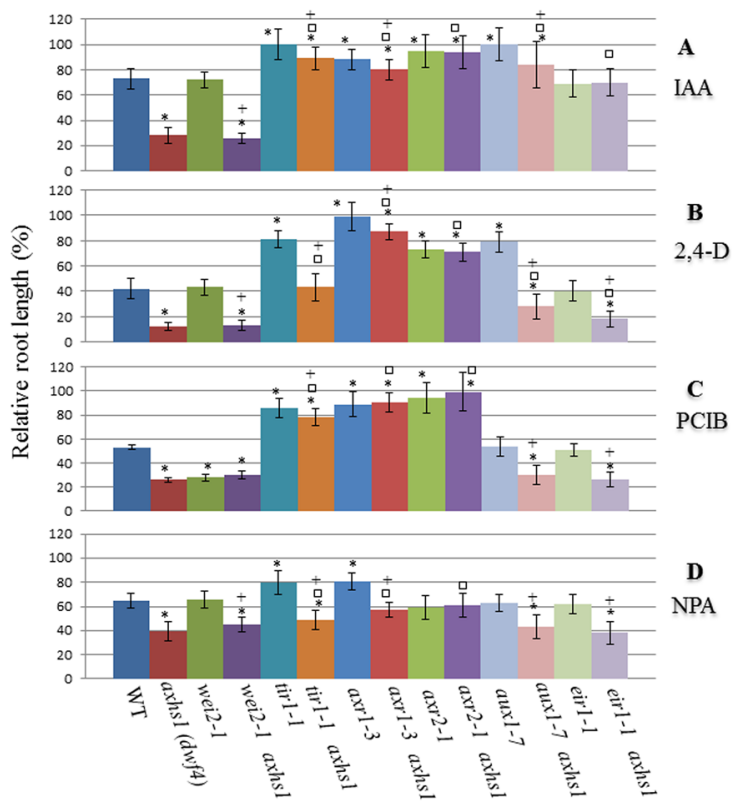

Figure 5. Interaction between AXHS1/DWF4 and genes involved in auxin biosynthesis, transport and signal transduction. Wild type, axhs1(dwf4), wei2-1, axhs1(dwf4) wei2-1, tir1-1, axhs1(dwf4) tir1-1, axr1-3, axhs1(dwf4) axr1-3, axr2-1, axhs1(dwf4) axr2-1, aux1-7, axhs1(dwf4) aux1-7, eir1-1, and axhs1(dwf4) eir1-1 seedlings were grown for 7 days in control medium and $60 \mathrm{nM}$ IAA, $60 \mathrm{nM}$ 2,4-D, $10 \mu \mathrm{M}$ PCIB, or $2.5 \mu \mathrm{M}$ NPA containing medium. Relative root length is expressed as a percentage of the length observed in unsupplemented medium. Data represent mean \pm SD of more than 20 seedlings from at least two independent experiments. ${ }^{*}=$ significant difference from wild type by Student's $t$-test $(p<0.05) . \square=$ significant difference from axhs $1 / d w f 4$ by Student's $t$-test $(p<0.05) .+=$ significant difference from wei2-1, tir1-1, axr1-3, axr2-1, aux1-7, or eir1-1 by Student's $t$-test $(p<0.05)$.
5). axhs1/dwf4 axr1-3 had an intermediate sensitivity to IAA, 2,4-D, and NPA between wild type and axr1-3, but it had levels of resistance to $10 \mu \mathrm{M}$ PCIB very similar to those of axr1-3 (Figure 5). On the other hand, with the exception of hypocotyl elongation, which was similar to that of the axhs1/dwf4 single mutant, the morphology of the axhs1/dwf4 axr1-3 mutant was indistinguishable from that of the axr1-3 single mutant (Figure 6 and Supplementary Figure S2). These results suggest that AXHS1/DWF4 functions at the level or downstream of AXR1.

As illustrated in Figure 5, tir1-1 exhibited resistance to IAA, 2,4-D, NPA, and PCIB in roots. The axhs1/dwf4 tir1-1 double mutant had an intermediate sensitivity to IAA, 2,4-D, NPA, and PCIB between axhs1/dwf4 and tir1-1 (Figure 5). axhs1/dwf4 tir1-1 appeared very similar to axhs1/dwf4 plants at seedlings and adult stage (Figure 6 and Supplementary Figure S2). Taken together, these results strongly suggest that $A X H S 1 / D W F 4$ functions at the level or downstream of TIR1.

Our results indicate that axr2-1 has increased resistance to PCIB, and a level of sensitivity to NPA similar to that of wild type roots (Figure 5). The axhs1/dwf4 axr2-1 mutant root growth exhibited levels of sensitivity to IAA, 2,4-D, PCIB, and NPA inhibition similar to those of the axr2-1 mutant (Figure 5). On the other hand, axhs1/dwf4 axr2-1 appeared very similar to axr2-1 plants at both seedling and adult stages (Figure 6 and Supplementary Figure S2). These results indicate that AXHS1/DWF4 acts upstream of AUX/IAA7 in the auxin signaling pathway.

The axhs1/dwf4 aux1-7 double mutant had intermediate sensitivity to IAA and 2,4-D between axhs1 and aux1-7 (Figure 5). In contrast, the level of sensitivity to NPA and PCIB in axhs1/dwf4 aux1-7 was very similar to that of the axhs1/dwf4 mutant (Figure 5).
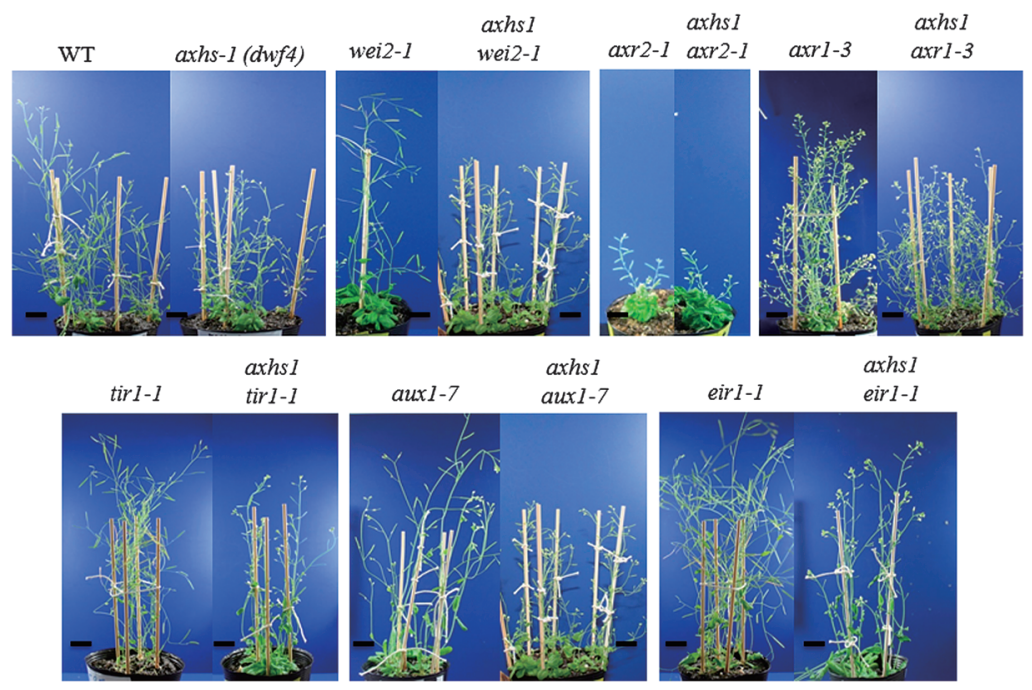

Figure 6. Phenotype of mature plants. Wild type and mutants plants were grown in soil for 6 weeks. Bars $=1 \mathrm{~cm}$. 


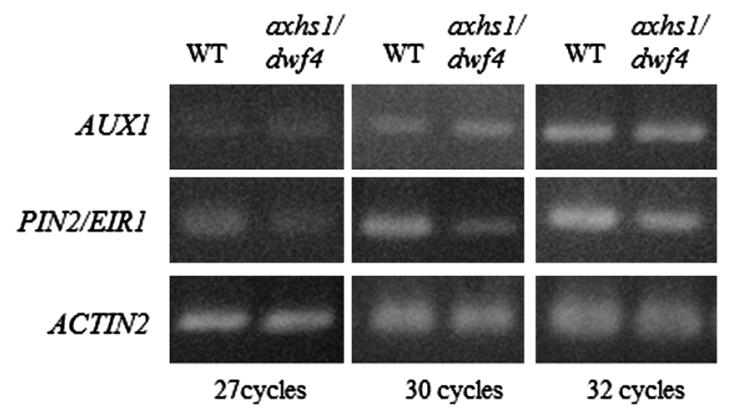

Figure 7. RT-PCR analysis. Seven-day-old wild type and axhs1/dwf4 seedlings were analyzed for expression of AUX1 and PIN2/EIR1. The number of PCR cycles is indicated. The figure illustrates representative results from three independent experiments.

These results indicate that AUX1 mediates IAA and 2,4-D hypersensitivity, but has no significant impact on NPA and PCIB hypersensitivity in axhs1/dwf4. On the other hand, the root growth of axhs1/dwf4 aux1-7 seedlings was similar to that of aux1-7, but they exhibited a short hypocotyl similar to that of axhs1/dwf4 (Supplementary Figure S2). The morphology of mature axhs1/dwf4 aux 1-7 plants was very similar to that of the axhs1/dwf4 single mutant (Figure 6).

The axhs1/dwf4 eir1-1 double mutant exhibited levels of sensitivity to 2,4-D, NPA and PCIB similar to those of the axhs1/dwf4 single mutant (Figure 5). In contrast, it displayed wild type sensitivities to IAA (Figure 5). These results indicate that PIN2/EIR1 mediates axhs1 hypersensitivity to IAA, but has no significant impact on the 2,4-D, NPA and PCIB sensitivity of the mutant. On the other hand, axhs1/dwf4 eir1-1 seedlings exhibited a short hypocotyl similar to that of axhs1, and a root growth similar to that of eir1-1 (Supplementary Figure S2). The morphology of axhs1 eir1-1 plants at adult stage was indistinguishable from that of the axhs1/dwf4 mutant (Figure 6).

In order to further evaluate the impact of the axhs1/dwf4 mutation on auxin transport, the expression of AUX1 and PIN2/EIR1 in the axhs1/dwf4 mutant was analyzed by RT-PCR analysis. The PIN2/EIR1 mRNA accumulation level was somewhat lower in axhs1/dwf4 than in wild type seedlings (Figure 7). In contrast, no significant differences were observed in the expression of AUX1 between axhs1/dwf4 and wild type seedlings (Figure 7).

\section{Auxin sensitivity of $B R$ mutants}

In order to further evaluate the cross talk between auxin and $\mathrm{BR}$ in controlling root elongation, we examined the growth of mutants affected in BR biosynthesis and BR signaling. BRI1 encodes a critical component of the BR receptor (Li and Chory 1997; Nam and Li 2002), and DWF6/DET2 encodes a putative steroid 5 alphareductase that catalyzes an early step of BR biosynthesis

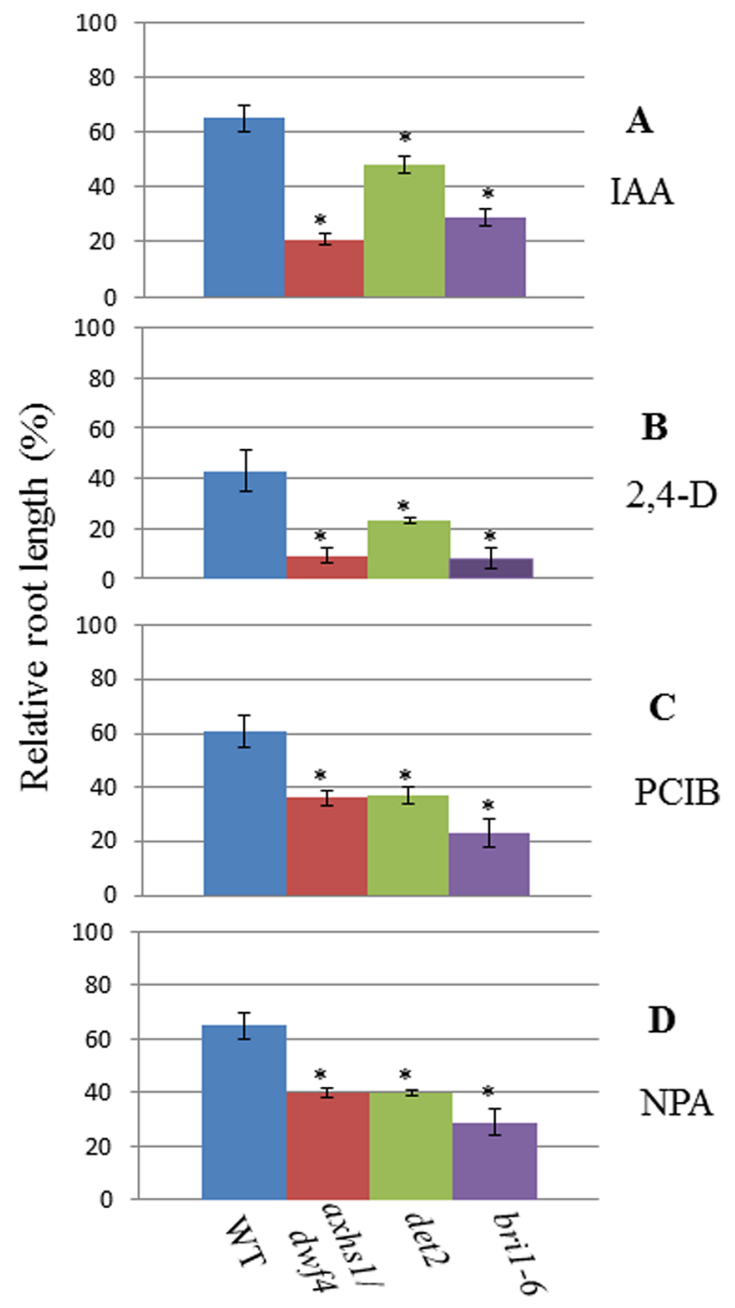

Figure 8. BR biosynthesis and BR signaling mutants are hypersensitive to auxins, antiauxins, and auxin transport inhibitors. Wild type, axhs1/dwf4, det2, and bri1-6 seedlings were grown for 7 days in medium supplemented with or without $60 \mathrm{nM}$ IAA, $60 \mathrm{nM}$ 2,4-D, $5 \mu \mathrm{M}$ PCIB, or $2.5 \mu \mathrm{M}$ NPA. Root length is expressed relative to untreated seedlings. Data are means $\pm \operatorname{SD}(n>20)$ from at least two independent experiments. An asterisk indicates a significant difference from wild type by Student's $t$-test $(p<0.05)$.

(Fujioka et al. 1997). As shown in Figure 8, axhs1/dwf4, det2 and bri1-6 showed enhanced inhibition of root growth in response to exogenous IAA, 2,4-D, PCIB and NPA. These results indicate that hypersensitivity to auxin and related compounds in roots is regulated by the BR biosynthesis and signal transduction pathways.

\section{Auxin-dependent gene expression in axhs 1/dwf4}

Our genetic interaction analyses indicate that the AXHS1/DWF4 mediated BR biosynthesis and the auxin signaling pathway cross-talk somewhere downstream of the site of AXR1-TIR1 action and somewhere upstream of the site of AXR2. Next, we analyzed the expression of the auxin-inducible reporter gene DR5:GUS in wild type and axhs1/dwf4 genotypes. The DR5:GUS reporter, which contains the AuxRE, is thought to reflect endogenous 

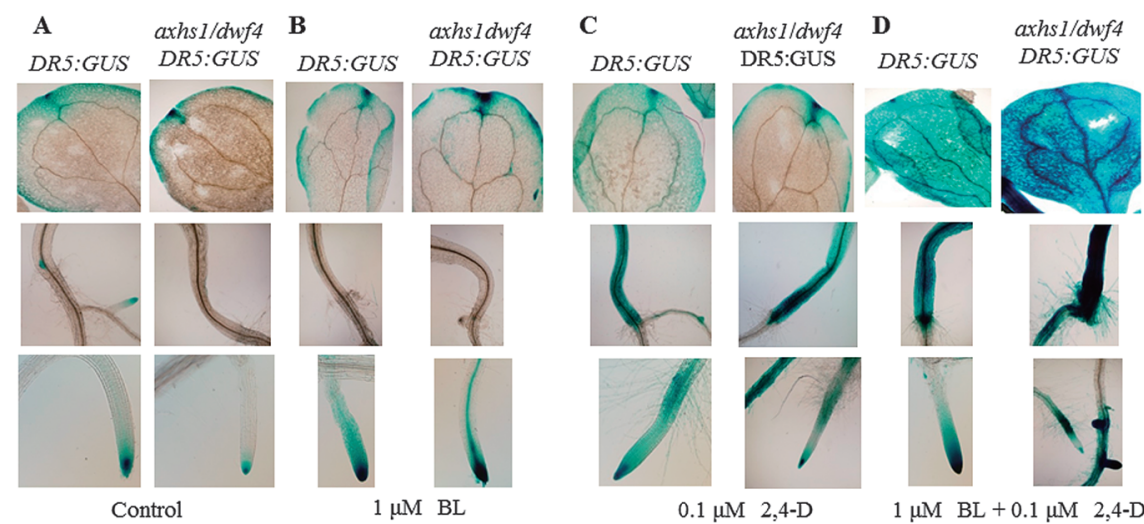

$0.1 \mu \mathrm{M} \quad 2,4-\mathrm{D}$

$1 \mu \mathrm{M}$ BL $+0.1 \mu \mathrm{M} \quad 2,4-\mathrm{D}$

Figure 9. Analysis of the expression of the auxin reporter DR5:GUS gene in wild type and axhs1/dwf4 mutant seedlings. GUS activity was analyzed in DR5:GUS and axhs1/dwf4 DR5:GUS seedlings grown in agar plates for 7 days and then incubated for $48 \mathrm{~h}$ in liquid medium (A), and liquid medium supplemented with either $1 \mu \mathrm{M}$ BL (B), $0.1 \mu \mathrm{M}$ 2,4-D (C), or $1 \mu \mathrm{M}$ BL and $0.1 \mu \mathrm{M}$ 2,4-D (D), respectively. Seedlings were stained for GUS for $20 \mathrm{~h}$, and representative seedlings from three independent experiments were photographed.

auxin distribution (Ulmasov et al. 1997). In order to generate axhs1/dwf4 DR5:GUS plants, we crossed a transgenic line harboring the DR5:GUS construct into the axhs1/dwf4 background. In axhs1/dwf4 seedlings grown in control medium, the expression of DR5:GUS expression was reduced compared to wild type seedlings (Figure 9A). In contrast, DR5:GUS and axhs1/dwf4 DR5:GUS seedlings treated with BL exhibited similar levels of GUS activity (Figure 9B), which indicates that BR promotes the expression of DR5:GUS. On the other hand, DR5:GUS expression was increased in wild type and axhs1/dwf4 seedlings treated with 2,4-D (Figure 9C). The expression of the reporter gene in axhs $1 / d w f 4$ seedlings treated with 2,4-D was reduced in cotyledons, but it was similar to wild type in roots and hypocotyls (Figure 9C). Interestingly, axhs1/dwf4 seedlings treated with both auxin and BR exhibited higher levels of DR5:GUS expression in cotyledons, hypocotyls, and roots compared to those of wild type seedlings (Figure 9D). These results indicate that the cellular balance of auxin and BR concentration may play an important role in the regulation of auxin-responsive genes.

\section{Auxin-dependent AUX/IAA protein degradation in axhs $1 / d w f 4$}

In order to evaluate AUX/IAA protein stability, we used transgenic plants carrying the HS:AXR3NT-GUS reporter gene, which encodes a heat shock-inducible fusion of the N-terminal portion of AUX/IAA17(AXR3) and GUS (Gray et al. 2001). We crossed the HS:AXR3NTGUS gene into the axhs1/dwf4 background and assessed protein stability in HS:AXR3NT-GUS and axhs1/dwf4 HS:AXR3NT-GUS lines (Figure 10). First, we did time course experiments to determine the level of AXR3NTGUS accumulation in wild type and axhs1/dwf4 seedlings treated with and without BR (Figure 10A, B). Seedlings of both genotypes were heat shocked for $2 \mathrm{~h}$ to promote AXR3NT-GUS gene expression, but the stability of the reporter protein in axhs1 was lower compared to that of wild type (Figure 10A, B). Increased levels of AXR3NT-GUS protein were observed in wild type and axhs1/dwf4 seedlings treated with BR (Figure 10A, B). Next, we analyzed the effect of auxin and BR interactions on AXR3NT-GUS protein stability (Figure 10C, D). 2,4-D promoted degradation of the reporter protein in wild type and axhs1/dwf4 seedlings. In contrast, BR prevented AXR3NT-GUS protein degradation in both wild type and axhs1/dwf4 seedlings treated with and without 2,4-D (Figure 10C, D). These results strongly suggest that BR inhibits auxin mediated degradation of AUX/IAA proteins.

\section{Discussion}

Isolation and characterization of the axhs 1 auxinhypersensitive mutant indicate the involvement of BR in auxin regulated root elongation. Results from BRfeeding experiments, Tail-PCR, DWF4 gene expression, and $d w f 4$ genetic complementation analyses indicated that axhs1 is a leaky mutation of DWF4 (Figure 3 and Figure 4). DWF4 is a C-22- $\alpha$-hydrolase that catalyzes the rate limiting step of BR biosynthesis (Choe et al. 1998). Null $d w f 4$ mutations (Azpiroz et al. 1998; Nakamoto et al. 2006) are infertile and showed a stronger dwarf phenotype compared to that of axhs1, indicating that axhs1 is a weak allele of DWF4.

Nakamoto et al. (2006) found that inhibition of BR biosynthesis by either a leaky mutation of DWF4 or Brz rescues defects in tropic responses of hypocotyls in the Arabidopsis nph4(arf7) mutant. In the present study, we show that the axhs1/dwf4 mutant is hypersensitive to auxins (IAA, 2,4-D), polar auxin transport inhibitors (TIBA, NPA), and inhibitors of the auxin-mediated degradation of AUX/IAA repressor proteins, such as PCIB. These phenotypes can be explained by a mutation affecting auxin biosynthesis, transport, or signaling 


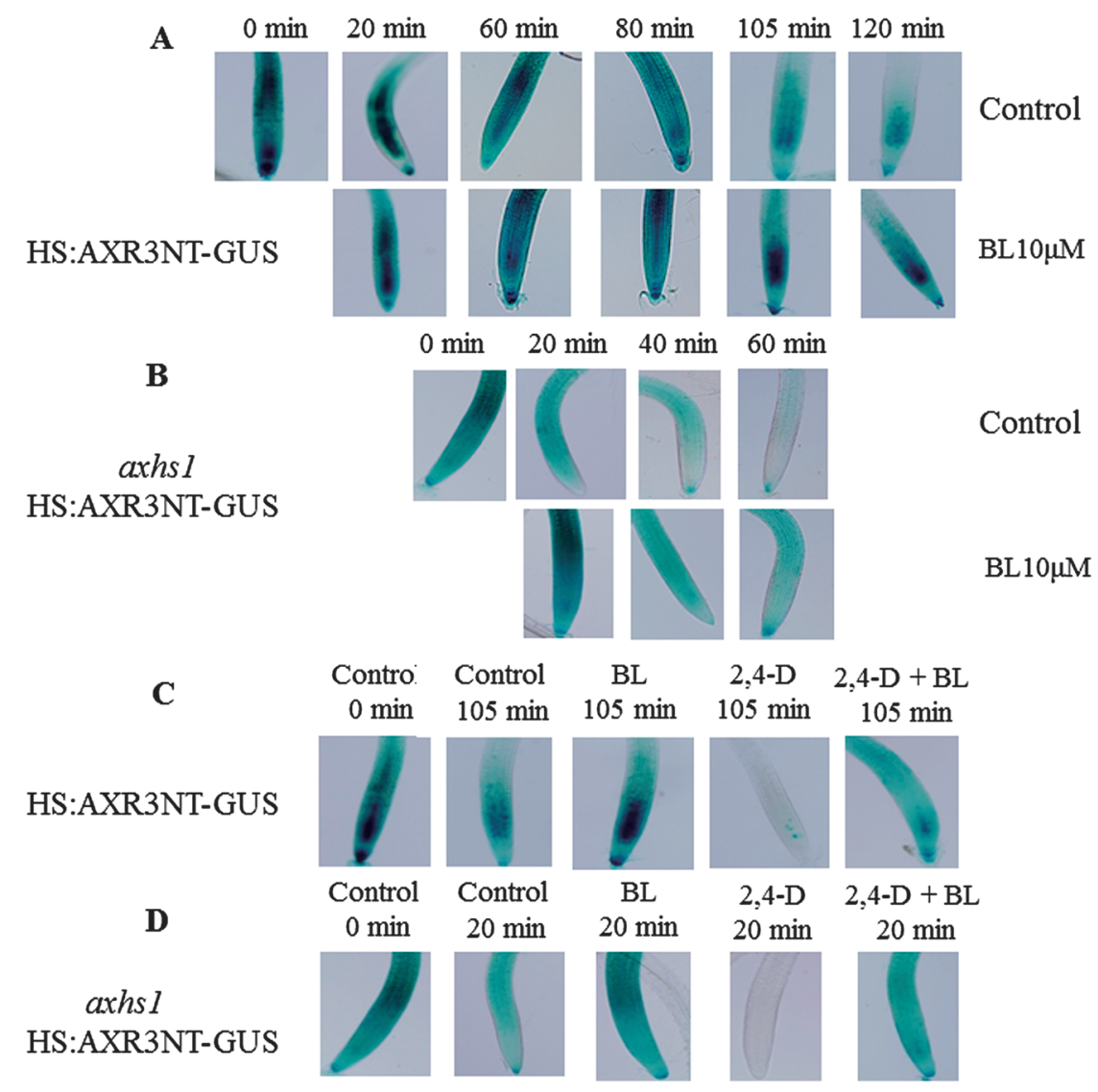

Figure 10. BR prevents the auxin-mediated degradation of an AUX/IAA reporter protein. Seven-day-old HS:AXR3NT-GUS (A) and axhs1/dwf4 HS:AXR3NT-GUS (B) seedlings were heat shocked at $37^{\circ} \mathrm{C}$ for $120 \mathrm{~min}$ to induce the expression of the fusion protein. After incubation at $23^{\circ} \mathrm{C}$ for $20 \mathrm{~min}$ (time 0 ), seedlings were transferred to new medium supplemented with or without $10 \mu \mathrm{M} \mathrm{BL}$, and incubated for the time periods listed. Sevenday-old HS:AXR3NT-GUS (C) and axhs1/dwf4 HS:AXR3NT-GUS (D) seedlings were treated as in (A, B) to induce the expression of the reporter protein. After incubation at $23^{\circ} \mathrm{C}$ for $20 \mathrm{~min}$, seedlings were mock-treated or treated with $10 \mu \mathrm{M} \mathrm{BL}, 5 \mu \mathrm{M} 2,4-\mathrm{D}$, and $10 \mu \mathrm{M}$ BL and $5 \mu \mathrm{M} 2,4-\mathrm{D}$ for the indicated periods of time. Seedlings were stained for $20 \mathrm{~h}$ to detect GUS activity. The figure illustrates representative results from three independent experiments.

pathway. Nakamura et al. (2003a) reported that the BR-deficient det2 mutant exhibited higher IAA levels compared to wild type plants, however, no change in the endogenous IAA levels per gram fresh weight was found following BR treatment of either wild type or $\operatorname{det} 2$ seedlings. In order to evaluate the impact of the axhs1/dwf4 mutation on auxin biosynthesis, we constructed and characterized a mutant deficient in both auxin and BR biosynthesis. ASA1 encodes the $\alpha$-subunit of anthranilate synthase, a rate limiting enzyme of Trp biosynthesis (Stepanova et al. 2005). The wei2-1 mutation had no significant impact on the axhs1/dwf4 phenotypes (Figure 5 and Figure 6). Taking into account that wei2-1 suppress the high-auxin phenotypes of sur1 and sur2, two-auxin overproducing mutants (Stepanova et al. 2005), our results strongly suggest that the axhs1 phenotypes are not caused by an increased amount of auxin in axhs1/dwf4. On the other hand, BR does not affect the expression of a ASA1:GUS reporter gene introduced into both axhs1/dwf4 and wild type genotypes (Supplementary Figure S3), which suggests that DWF4 does not have a significant role in regulating the ASA1-mediated auxin biosynthesis pathway. In contrast, it has been reported that auxin stimulates DWF4 expression and BRs biosynthesis through the auxin-signaling pathway (Chung et al. 2011; Yoshimitsu et al. 2011).

Since auxin polar transport inhibitors such as NPA and TIBA have been proposed to affect root growth by increasing auxin levels in the root tip, hypersensitivity could result from a change in auxin transport or an increased auxin response in the axhs1/dwf4 mutant. Thus, mutants such as tir 3 where resistant to NPA and had clear defects in auxin transport (Ruegger et al. 1997), and mutants such as tirl were defective in auxin response (Ruegger et al. 2012). Our pharmacological and genetic analyses indicate that AXHS1/DWF4 is involved in both auxin signaling and auxin uptake. The axhs1/dwf4 eir1-1 double mutant had a level of sensitivity to 2,4-D similar to that of the axhs1/dwf4 single mutant, whereas it displayed a root growth on IAA similar to that of wild type seedlings (Figure 5). Because IAA and NAA are 
preferentially transported out of the cells by auxin efflux carriers such as PIN2/EIR1, while 2,4-D largely diffuses (Delbarre et al. 1996; Luschnig et al. 1998), our results indicate that a reduction of the PIN2/EIR1 mediated auxin transport may rescue the IAA-hypersensitive phenotype in axhs1/dwf4. RT-PCR analysis indicated that the expression of PIN2/EIR1 is somewhat reduced in axhs1/dwf4 compared to wild type seedlings (Figure 7), which suggests that BR deficiency caused by axhs $1 / d w f 4$ leads to reduction of PIN2/EIR1-mediated polar auxin transport. Consistent with the above-indicated results, it has been shown that BRs interact with auxin to promote lateral root development and stimulate plant tropisms in roots and hypocotyls through activation of polar transport of auxin in root and shoot (Bao et al. 2004; Li et al. 2005). Li et al. (2005) reported that the expression of PIN1 and PIN2/EIR1 genes was induced in wild type plants by BR treatment, whereas it was suppressed in BR biosynthesis-deficient mutants such as $\operatorname{det} 2$ and $\operatorname{dim} 1$. In addition, BR induced the basipetal accumulation of PIN2/EIR1 and stimulated the extended localization of the protein into the root elongation zone in response to gravity (Li et al. 2005). Taking into account that AUX1 is not expressed in root cortical cells (Swarup et al. 2001, 2004), there is the possibility that defects in PIN2/EIR1 protein localization in root epidermal and cortical cells will result in an altered distribution of auxin between cells of the meristem and root elongation zones in axhs $1 / d w f 4$.

aux1 mutations disrupt IAA and 2,4-D, but not NAA accumulation (Marchant et al. 1999; Rahman et al. 2001; Swarup et al. 2004). The axhs1/dwf4 aux1-7 double mutant had an intermediate sensitivity to 2,4-D and IAA between axhs1/dwf4 and aux1-7 (Figure 5). In contrast, axhs1/dwf4, aux1-7, and axhs1/dwf4 aux1-7 mutants exhibited wild type levels of sensitivity to the lipophilic synthetic auxin NAA (Figure 2, data not shown). These results indicate that the IAA and 2,4-D hypersensitivity of axhs1/dwf4 is mediated by an AUX1 dependent auxin uptake, and suggest that auxin influx is accelerated leading to the increase of local concentrations of auxin in the axhs1/dwf4 mutant. RT-PCR analysis indicated that the level of expression of AUX1 was similar in wild type and axhs1/dwf4 seedlings (Figure 7), however, defects in AUX1 protein localization might account for an increased auxin influx in axhs1/dwf4.

Nakamura et al. (2006) reported that Aux/IAA proteins may function as signaling components modulating auxin and BR responses in an organdependent manner (Nakamura et al. 2006). Our results indicate that mutants affected in auxin signal transduction (axr1-3, axr2-1, tir1-1) were resistant to the antiauxin PCIB, while those affected in auxin transport (aux1-7, eir1-1) exhibited wild type sensitivity to that antiauxin (Figure 5). Thus, auxin and PCIB hypersensitivity could be explained by an increased auxin response in the axhs1/dwf4 mutant. $\operatorname{det} 2$ and bri1-6 mutants were also hypersensitive to auxins and the antiauxin PCIB (Figure 8), which suggests that homeostatic control of BR signaling is required for a normal response to auxin. On the other hand, genetic interaction analyses strongly suggest that AXHS1/ DWF4 and the auxin signaling pathway cross-talk somewhere downstream of the site of AXR1-TIR1 action and somewhere upstream of the site of AXR2 (AUX/IAA7) action (Figure 5). Moreover, the results of our AXR3NT-GUS protein stability assays suggest that BR prevents the auxin-dependent degradation of the AUX/IAA17 (AXR3) protein through the ubiquitinproteasome pathway. Nemhauser et al. (2004) reported that treatment with $1 \mu \mathrm{M}$ BR does not induce AXR3NTGUS protein turnover in wild type seedlings. They did time course experiments, but they did not do extensive quantification of the later time points ( 80 and $150 \mathrm{~min}$ after the end of the heat shock period), where, judging from the published images, increased stability of the reporter would be detected (Nemhauser, personal communication). We did time course experiments to analyze the effects of $10 \mu \mathrm{M}$ BR on AXR3NT-GUS protein degradation in wild type and axhs1/dwf4 roots (Figure 10A, B). After that, we focused on BR concentration: a) wild type BR levels, b) reduced BR levels (axhs1/dwf4 seedlings), c) increased BR levels (treatment with $10 \mu \mathrm{M} \mathrm{BR}$ ). Our results indicate that BR prevents AXR3NT-GUS protein degradation in both wild type and axhs1 mutant seedlings treated with and without $5 \mu \mathrm{M}$ 2,4-D (Figure 10C, D).

DR5:GUS expression analyses (Figure 9) indicate that the expression of DR5:GUS was reduced in the axhs1/dwf4 mutant, but rescued by BR administration, suggesting that BRs are essential for full induction of the reporter gene. BR-treated seedlings showed higher levels of and broader DR5:GUS expression from the root tip to the elongation zone (Figure 9). Besides, GUS expression in axhs $1 / d w f 4$ seedlings treated with exogenous auxin was reduced in cotyledons, but it was similar to wild type in roots and hypocotyls (Figure 9). Since the axhs1/dwf4 mutation inhibits the actions of exogenously applied auxin, results indicate that AXHS1/DWF4 may function downstream of auxin and somewhere upstream of DR5:GUS. Interestingly, axhs1/dwf4 seedlings treated with both auxin and BR exhibited higher levels of DR5:GUS expression in cotyledons, hypocotyls, and roots compared to those of wild type seedlings (Figure 9).

Results from our pharmacological, genetic interaction, DR5:GUS expression, and AXR3NTGUS protein degradation analyses can be explained by a BR-deficiency activation of auxin signaling, but not just by an accelerated auxin influx leading to the increase of intracellular auxin in root apical tissues of 
axhs1/dwf4. Collectively, the data indicate that is not the absolute level of auxin and BR concentration, but the concentration ratio that may play an essential role in the regulation of auxin and BR mediated responses, such as root elongation. The complex biological significance of this mechanism of interaction between auxin and BR should be determined in further studies.

\section{Acknowledgements}

We would like to thank Dr. Satomi Takeda, and members of the Sekiya, Takase, and Prieto laboratories for technical and intellectual input throughout the course of this research.

\section{References}

Alonso JM, Stepanova AN, Leisse TJ, Kim CJ, Chen H, Shinn P, Stevenson DK, Zimmerman J, Barajas P, Cheuk R, et al. (2003) Genome-wide insertional mutagenesis of Arabidopsis thaliana. Science 301: 653-657

Azpiroz R, Wu Y, LoCascio JC, Feldman KA (1998) An Arabidopsis brassinosteroid-dependent mutant is blocked in cell elongation. Plant Cell 10: 219-230

Bao F, Shen J, Brady SR, Muday GK, Asami T, Yang Z (2004) Brassinosteroid interact with auxin to promote lateral root development in Arabidopsis. Plant Physiol 134: 1624-1631

Choe S, Dilkes BP, Fujioka S, Takatsuto S, Sakurai A, Feldman KA (1998) The DWF4 gene of Arabidopsis encodes a cytochrome P450 that mediates multiple $22 \alpha$-hydroxylation steps in brassinosteroid biosynthesis. Plant Cell 10: 231-243

Chung Y, Maharjan PM, Lee O, Fujioka S, Jang S, Kim B, Takatsuto S, Tsujimoto M, Kim H, Cho S, et al. (2011) Auxin stimulates DWARF4 expression and brassinosteriod biosynthesis in Arabidopsis. Plant J 66: 564-578

Delbarre A, Muller P, Imhoff V, Guern J (1996) Comparison of mechanisms controlling uptake and accumulation of 2,4-dichloro-phenoxy acetic acid, naphthalene-1-acetic acid, and indole-3-acetic acid in suspension-cultured tobacco cells. Planta 198: $532-541$

Fujioka S, Li J, Choi YH, Seto H, Takatsuto S, Noguchi T, Watanabe T, Kuriyama H, Yokota T, Chory J, et al. (1997) The Arabidopsis deetiolated 2 mutant is blocked early in brassinosteroid biosynthesis. Plant Cell 9: 1951-1962

Goda H, Sawa S, Asami T, Fujioka S, Shimada Y, Yoshida S (2004) Comprehensive comparison of auxin-regulated and brassinosteroid-regulated genes in Arabidopsis. Plant Physiol 134: $1555-1573$

Gray WM, Kepinski S, Rouse D, Leyser O, Estelle M (2001) Auxin regulates SCFTIR1-dependent degradation of AUX/IAA proteins. Nature 414: 271-276

Haughn GW, Somerville C (1986) Sulfonylurea-resistant mutants of Arabidopsis thaliana. Mol Gen Genet 204: 430-434

Hayashi KI, Jones AM, Ogino K, Yamazoe A, Oono Y, Inouchi M, Kondo H, Nozaki H (2003) Yokonolide B, a novel inhibitor of auxin action, blocks degradation of AUX/IAA factors. J Biol Chem 278: 23797-23806

Lau S, Jurgens G, De Sme ID (2008) The evolving complexity of the auxin pathway. Plant Cell 20: 1738-1746

Leyser HM, Lincoln CA, Timpte C, Lammer D, Turner J, Estelle M (1993) Arabidopsis auxin-resistance gene AXR1 encodes a protein related to ubiquitin-activating enzyme E1. Nature 364:
$161-164$

Li J, Chory J (1997) A putative leucine-rich repeat receptor kinase involved in brassinosteroid signal transduction. Cell 90: 929-938

Li L, Xu J, Xu ZH, Xue HW (2005) Brassinosteroid stimulate plant tropisms thorough modulation of polar auxin transport in Brassica and Arabidopsis. Plant Cell 17: 2738-2753

Liu YG, Mitsukawa N, Oosumi T, Whittier RF (1995) Efficient isolation and mapping of Arabidopsis thaliana T-DNA insert junctions by thermal asymmetric interlaced PCR. Plant $J$ 8: 457-462

Luschnig C, Gaxiola RA, Grisafi P, Fink GR (1998) EIR1, a rootspecific protein involved in auxin transport, is required for gravitropism in Arabidopsis thaliana. Genes Dev 12: 2175-2187

Marchant A, Kargul J, May ST, Muller P, Delbarre A, PerrotRechenmann C, Bennet MJ (1999) AUX1 regulates root gravitropism in Arabidopsis by facilitating auxin uptake within root apical tissues. EMBO J 18: 2066-2073

Marchant A, Bhalerao R, Casimiro I, Eklof J, Casero PJ, Bennet MJ, Sandberg G (2002) AUX1 promotes lateral root formation by facilitating indole-3-acetic-acid distribution between sink and source tissues in the Arabidopsis seedling. Plant Cell 14: 589-597

Mashiguchi K, Tanaka K, Sakai T, Sugawara S, Kawaide H, Natsume M, Hanada A, Yaeno T, Shirasu K, Yao H, et al. (2011) The main auxin biosynthesis pathway in Arabidopsis. Proc Natl Acad Sci USA 108: 18512-18517

Muller A, Guan C, Galweiler L, Tanzler P, Huijser P, Marchant A, Parry G, Bennett M, Wisman E, Palme K (1998) AtPIN2 defines a locus of Arabidopsis for root gravitropism control. EMBO J 17: 6903-6911

Nagpal P, Walker LM, Young JC, Sonawala A, Timpte C, Estelle M, Reed JW (2000) AXR2 encodes a member of the Aux/IAA protein family. Plant Physiol 123: 563-573

Nakamoto D, Ikeura A, Asami T, Yamamoto K (2006) Inhibition of brassinosteroid biosynthesis by either a dwarf4 mutation or a brassinosteroid biosynthesis inhibitor rescues defects in tropic responses of hypocotyls in the Arabidopsis mutant Nonphototropic hypocotyl 4. Plant Physiol 141: 456-464

Nakamura A, Higuchi K, Goda H, Fujiwara MT, Sawa S, Koshiba T, Shimada Y, Yoshida S (2003a) Brassinolide induces IAA5, IAA19, and DR5, a synthetic auxin response element in Arabidopsis, implying a cross talk point of brassinosteroid and auxin signaling. Plant Physiol 133: 1843-1853

Nakamura A, Nakajima N, Goda H, Shimada Y, Hayashi K, Nozaki H, Asami T, Yoshida S, Fujioka S (2006) Arabidopsis Aux/IAA genes are involved in brassinosteroid-mediated growth responses in a manner dependent on organ type. Plant J 45: 193-205

Nakamura A, Shimada Y, Goda H, Fujiwara MT, Asami T, Yoshida $S$ (2003b) AXR1 is involved in BR-mediated elongation and SAUR-AC1 gene expression in Arabidopsis. FEBS Lett 553: 28-32

Nam KH, Li J (2002) BRI1/BAK1, a receptor kinase pair mediating brassinosteroid signaling. Cell 102: 203-212

Nemhauser JL, Mokler TC, Chory J (2004) Interdependency of brassinosteroid and auxin signaling in Arabidopsis. PlosS Bio 2: E258

Oono Y, Ooura C, Rahman A, Aspuria ET, Hayashi K, Tanaka A, Uchimiya H (2003) p-Chlorophenoxyisobutiric acid impairs auxin response in Arabidopsis root. Plant Physiol 133: 1135-1147

Rahman A, Ahamed A, Amakawa T, Goto N, Tsurumi S (2001) Chromasaponin I specifically interacts with AUX1 protein in regulating the gravitropic response of Arabidopsis roots. Plant Physiol 125: 990-1000

Rahman A, Bannigan A, Sulaman W, Pechter P, Blancaflor EB, 
Baskin TI (2007) Auxin, actin and growth of the Arabidopsis thaliana primary root. Plant J 50: 514-528

Rahman A, Takahashi M, Shibasaki K, Wu S, Inaba T, Tsurumi S, Baskin TI (2010) Gravitropism of Arabidopsis thaliana roots requires the polarization of PIN2 toward the root tip in meristematic cortical cells. Plant Cell 22: 1762-1776

Ruegger M, Dewey E, Gray WM, Hobbie L, Turner J, Estelle M (2012) The TIR1 protein of Arabidopsis functions in auxin response and is related to human SKP2 and yeast Grr1p. Genes Dev 12: 198-207

Ruegger M, Dewey E, Hobbie L, Brown D, Benasconi P, Turner J, Muday G, Estelle M (1997) Reduced NPA-binding in the tir3 mutant of Arabidopsis is associated with a reduction of polar auxin transport and diverse morphological defects. Plant Cell 9: 745-757

Stepanova AN, Hoyt JM, Hamilton AA, Alonso JM (2005) A link between ethylene and auxin uncovered by the characterization of two root-specific ethylene-insensitive mutants in Arabidopsis. Plant Cell 17: 2230-2242

Swarup R, Friml J, Marchant A, Ljung K, Sandberg G, Palme K, Bennet M (2001) Localization of the auxin permease AUX1 suggests two functionally distinct hormone transport pathways operate in the Arabidopsis root apex. Genes Dev 15: 2648-2653

Swarup R, Kargul J, Marchant A, Zadik D, Rahman A, Mils R, Yemm A, May S, Williams L, Millner P, et al. (2004) Structurefunction analysis of the presumptive Arabidopsis auxin permease AUX1. Plant Cell 16: 3069-3083

Ulmasov T, Murfett J, Hagen G, Guilfoyle TJ (1997) Aux/IAA proteins repress expression of reporter genes containing natural and highly active synthetic auxin response elements. Plant Cell 9: 1963-1971

Yoshimitsu Y, Tanaka K, Fukuda W, Asami T, Yoshida S, Hayashi K, Kamiya Y, Jikumaru Y, Shigeta T, Nakamura Y, et al. (2011) Transcription of DWARF4 plays a crucial role in auxin-regulated root elongation in addition to brassinosteroid homeostasis in Arabidopsis thaliana. PlosS Bio 6: E23851

Woodward AW, Bartel B (2005) Auxin: regulation, action, and interaction. Ann Bot (Lond) 95: 707-735

Wu G, Lewis DR, Spalding P (2007) Mutations in Arabidopsis multidrug resistance-like $\mathrm{ABC}$ transporters separate the roles of acropetal and basipetal auxin transport in lateral root development. Plant Cell 19: 1826-1837 